\title{
Prenylcysteine oxidase 1, a pro-oxidant enzyme of low density lipoproteins
}

Luis V. Herrera-Marcos, ${ }^{1,2}$ Jose M. Lou-Bonafonte, ${ }^{2,3,4}$ Maria V. Martinez-Gracia, ${ }^{4}$ Carmen Arnal, ${ }^{2,4,5}$, Maria A. Navarro ${ }^{1,2,4}$, Jesus Osada ${ }^{1,2,4}$

${ }^{1}$ Departamento de Bioquimica y Biologia Molecular y Celular, Facultad de Veterinaria, Instituto de Investigacion Sanitaria de Aragon-Universidad de Zaragoza, Zaragoza, E-50013, Spain, ${ }^{2}$ Instituto Agroalimentario de Aragon, CITA-Universidad de Zaragoza, Spain, ${ }^{3}$ Departamento de Farmacologia y Fisiologia, Facultad de Ciencias de la Salud y del Deporte, Instituto de Investigacion Sanitaria de Aragon-Universidad de Zaragoza, Huesca, E-22002, Spain, ${ }^{4}$ CIBER de Fisiopatologia de la Obesidad y Nutricion, Instituto de Salud Carlos III, Madrid, E-28029, Spain, ${ }^{5}$ Departamento de Patologia Animal, Facultad de Veterinaria, Instituto de Investigacion Sanitaria de Aragon-Universidad de Zaragoza, Zaragoza, E-50013, Spain,

\section{TABLE OF CONTENTS}

1. Abstract

2. Introduction

3. Human PCYOX1 gene

3.1. Gene polymorphisms

3.2. Hepatic PCYOX1 transcripts

3.3. Hepatic $P C Y O X 1$ transcriptional regulation

3.4. Hepatic PCYOX1 post-transcriptional regulation

4. PCYOX1 Protein

4.1. Post-translational modifications (PTMs)

4.2. Protein structure and function

4.3. Protein interactions

5. PCYOX1 in physiology and disease

5.1. Lipoproteins

5.2. Pcyox1-deficient mice

5.3. PCYOX1 and the liver

5.4. PCYOX1 gene and cancer

5.5. PCYOX1 and neurodegenerative diseases

6. Conclusions

5.6. PCYOX1 and obesity

7. Acknowledgments

8. References

\section{ABSTRACT}

Elevated levels of low density lipoproteins (LDLs) cause atherosclerotic disease, and proteomic analyses have found that these lipoproteins are endowed with prenylcysteine lyase. This systematic review summarizes current understanding of this enzyme, now known as prenylcysteine oxidase 1 (PCYOX1), which hydrolyzes the thioether bond of prenylcysteines in the final step in the degradation of prenylated proteins, releasing hydrogen peroxide, cysteine and the isoprenoid aldehyde. Despite the high variability of the PCYOX1 gene, no polymorphism has yet been associated with any disease. The liver, which is responsible for vehiculization of the enzyme in lipoproteins, is one of the main organs responsible for its expression, together with the gastrointestinal tract, kidney, male reproductive tissue and muscle. Moreover, although hepatic mRNA expression is sensitive to diet and hormones, the repercussion of these changes in LDLs containing PCYOX1 has not been addressed. One consequence of its elevated activity could be an increase in hydrogen peroxide, which might help to propagate the oxidative burden of LDLs, thus making PCYOX1 a potential pharmacological target and a new biomarker in cardiovascular disease.

\section{INTRODUCTION}

Lipids play a crucial role in organ homeostasis and, given their insolubility in the aqueous environment of plasma, require a sophisticated lipoprotein transport mechanism to move between tissues. Plasma lipoproteins can be separated into chylomicrons, very low-density lipoproteins (VLDLs), low-density lipoproteins (LDLs) and high-density lipoproteins (HDLs), in order of increasing floating density, by ultracentrifugation. VLDLs, which are initially assembled in the endoplasmic reticulum of hepatocytes and further lipidated in the Golgi, are secreted into the circulation. VLDLs contain apolipoprotein B100 (APOB100) as the structural apolipoprotein and acquire other apolipoproteins (APOA1, APOA2, APOA4, APOC1, APOC2, APOC3 or APOE) once in the plasma. VLDLs transport triacylglycerols (TGs) from the liver to peripheral tissues, where lipoprotein lipase hydrolyzes the TGs to release non-esterified fatty acids for use as an energy source, leaving behind VLDL remnant particles. When these remnants are hydrolyzed by hepatic lipase, the resulting particles are known as LDLs. The latter, which are particularly enriched in cholesterol, are subsequently internalized by interaction with the LDL receptor and provide cholesterol to build membranes and to biosynthesize steroid hormones in peripheral tissues (1). Despite these physiological roles, a large 
number of studies have proved that elevated levels of LDLs cause atherosclerotic cardiovascular disease (2) and should therefore be a treatment target (3).

The fact that the non-APOB proteins present in LDL might modulate interactions with other proteins has prompted a series of proteomic analyses aimed at characterizing these minority proteins and their potential role in promoting or retarding LDL atherogenicity (4). Thus, using liquid isoelectrofocusing and polyacrylamide electrophoresis together with mass spectrometry, Banfi et al. proved that LDLs contain previously described apolipoproteins such as APOB100, APOA1, APOA4, APOE, APOJ and APOH. These authors also identified new proteins such as APOM, APOD, orosomucoid, retinol binding protein, paraoxonase 1 and prenylcysteine lyase (5).

Prenylcysteine lyase (PCL1), the current recommended name of which is prenylcysteine oxidase 1 (PCYOX1), is also known as KIAA0908 or UNQ597/PRO1183. This enzyme is able to cleave the thioether bond of prenylcysteines in prenylated proteins, releasing a free cysteine and the aldehyde of the isoprenoid in what could be the final step in the degradation of prenylated proteins $(6,7)$. According to the International Union of Biochemistry and Molecular Biology, it is classified as an oxidoreductase that acts on donors containing a sulfur group, with oxygen being the acceptor (EC 1.8.3.5.) (8). Prenylated proteins may contain either a 15-carbon isoprenoid residue (farnesyl) or its 20-carbon counterpart (geranylgeranyl) covalently attached to cysteines located at or near their carboxyl end. Studies with the purified bovine enzyme showed that both farnesylcysteine and geranylgeranylcysteine can act as substrates (6). Prenylated proteins represent $2 \%$ of all cellular proteins, therefore an enzyme that is able to remove the isoprenoid residues in mammalian cells may play an important role in their normal turnover and open up the possibility of better characterizing their regulation. Moreover, the presence of this protein in plasma lipoproteins $(5,9)$ has increased interest in it, thus meaning that its wide spectrum of action and its poorly known regulation warrant further research in the near future.

Although PCYOX1 was first discovered in 1997 (6), very little information has been published since then. The present report adheres to systematic review guidelines (10) and data were collected according to the criteria shown in Figure 1. The search in Pubmed (http://www.ncbi.nlm.nih.gov/pubmed/) using the Key Words (PCYOX1 and PCL1) identified 19 hits between November 1945 and 08 June 2017. An additional search in electronic databases was also carried out. The combined information from both sources formed the basis of this review.

\section{HUMAN PCYOX1 GENE}

The PCYOX1 gene is located in the forward strand of chromosome 2, specifically in the 2p13.3. position, and has a length of 23,805 base pairs (bp). Classically, it was thought to contain six exons and five introns (11-13), although new highthroughput technologies of RNAseq have identified three additional exons (14).

\subsection{Gene polymorphisms}

A total of 1610 single nucleotide polymorphisms (SNPs) have been found for the PCYOX1 gene to date, with 387 of these in the protein coding DNA (12). As can be seen from Table 1, the majority of SNPs in this particular region correspond to missense variations and coding sequence variants. No experimental evidence has been provided to establish the pathological significance of those variants described by the Exome Aggregation Consortium. Other sources of genomic variability present in this gene include copy number variations (21), short tandem repeats (19), inversions (2) and insertions (7) (16). Only four copy variations (nsv2776363, nsv2775326, nsv2772356, nsv533349) have been found to be associated with cytogenetics abnormalities, and no further validation has been carried out to assess their pathological contribution. Whether the high variability found in this gene in population studies translates into activity, and its consequences as regards the properties of LDLs, remains an open question at this stage.

\subsection{Hepatic PCYOX1 transcripts}

Since hepatic expression is responsible for the vehiculization of PCYOX1 in lipoproteins, only the expression of this organ will be discussed herein. However, this gene shows a nearly ubiquitous expression pattern (Figure 2), with the endocervix and testicles being the highest and the lowest expressing tissues, respectively (14).

In the liver (Figure 3A), the PCYOX1 gene encodes for eight transcripts (14) generated by five different transcription start sites and alternative splicing. Only five of these codify for a protein (17). PCYOX1-001 has a length of 5345 bp distributed over six exons. Its open reading frame is $1515 \mathrm{bp}$ and generates the isoform 1 of 505 amino acids, which is recognized as a canonical sequence (18). PCYOX1-002 has a length of 2959 bp distributed over six exons and generates a protein of 293 amino acids. PCYOX1-003 has a length of 556 bp distributed over four exons and generates a protein of 129 amino acids. PCYOX1-004 has a length of $648 \mathrm{bp}$ distributed over four exons and generates a protein of 154 amino acids. PCYOX1-006 has a length of 957 bp distributed over five exons and generates a protein of 209 amino acids. No annotation has been given to two transcripts found by next generation sequencing, although they could also be translated into protein since they use the transcription start site of transcripts PCYOX1-004 and PCYOX1-006. A Western analysis using high sensitive detection revealed the hepatic expression of those predicted proteins in mice (Figure 3B). PCYOX1-001, PCYOX1-002 and PCYOX1-006 showed higher molecular masses 
than expected based on their amino acid numbers, although this was not the case for PCYOX1-003 and PCYOX1-004. Future research will be required to establish the role of these isoforms in hepatic metabolism, and which of them is carried out by LDLs.

\subsection{Hepatic PCYOX1 transcriptional regulation}

Once again, the information regarding the liver will be considered using the HepG2 cell line as one of the most widely studied for this purpose. As mentioned above, the PCYOX1 gene has five potential transcription start sites. Four TATA-boxes, located in positions $(-701 .-685,-661 .-645,-638 .-615$ and -450. -434) (19), could be used by RNA polymerase to start transcription. According to the data retrieved from the Genome server from UCSC (20), and shown in Figure 4, there is a high frequency of repetitive elements (long interspersed nuclear elements (LINEs), short interspersed nuclear elements (SINEs) and retrotransposons (LTRs)) throughout the gene. The regulatory mechanisms of these elements are totally unknown.

Using the ENCODE database (21), the information in which is based on experimental observations, eight transcription factors modulating PCYOX1 expression in HepG2 were found (see Table 2). Most of these participate in general cell processes, modulating a wide range of transcription factors, and have ubiquitous expression $(14,22)$. As shown in Figure 5A, they bind to a region found in the third intron. A closer observation of the third intron (Figure 5B) reveals that all of these bind within a region spanning $400 \mathrm{bp}(70,497,100-70,496,700$ of chromosome 2). Despite the breakthrough that represents the ENCODE project, it remains difficult to offer a complete picture of the transcription factors that may be recruited to this gene under numerous biological circumstances.

To investigate the changes in PCYOX1 hepatic expression inadvertently reported by different authors using transcriptomic analyses, a search for these changes was carried out in the publically available Genome Expressed Omnibus (23). The results are summarized in Table 3. As can be seen, the mRNA levels of this gene were increased in mice lacking caspase 1, NADH-cytochrome P450 reductase, stearoyl-coA desaturase, SIRT1, glycerol kinase, retinoblastoma protein, Mdr2, Dicer1, in a model of progeria and in mice overexpressing lipin-1 beta. In contrast, the absence of Pdss2, Il4 and Il-13, suppressor of cytokine signaling 3, HNF4A or GBA decreased Pcyox1 expression. Some dietary conditions, such as depletion of polyunsaturated fatty acids or dietary palatinose, also decreased its expression, while a ketogenic diet increased it. Likewise, an increase was reported in concanavalin-induced hepatitis, although alcohol-induced hepatitis had the opposite effect. A decreased expression was found following administration of glucocorticoids, perfluorooctanoic acid or an increase in hepatic Pcyox1 hormone expression. Using Apoe-deficient mice, we did not observe any changes upon feeding a diet containing nuts (24) or following squalene administration to wild-type mice (25). These findings indicate that hepatic Pcyox1 expression has a complex effect in terms of expression regulation, that diet and hormones are important elements, and that the effect of these changes in lipoproteins requires future research.

\subsection{Hepatic PCYOX1 post-transcriptional regulation}

Bioinformatics tools allow interactions of the murine Pcyox1 gene with microRNA to be predicted (26). According to the obtained results, 13 miRNAs showed a high score for this interaction. Of these thirteen putative miRNAs, only Mir124a-2, Mir3474, Mir5112 and Mir3473d were found in the liver at a low level of expression. The presence of hepatic miRNAs regulating translation of Pcyox 1 mRNA adds a new level of complexity to this regulation and widens PCYOX1 levels in lipoproteins when those miRNA might undergo changes.

\section{HEPATIC PCYOX1 PROTEIN}

As already mentioned, the canonical PCYOX1-001 mRNA codes for a 505 amino acid protein. This 505-amino acid flavin adenine dinucleotide (FAD)-dependent thioether monooxygenase was found to have a molecular mass of $63 \mathrm{kDa}$ when first isolated from bovine brain membranes (6), with the same size being found in mouse liver (Figure 3B). In contrast to mRNA expression levels, the liver is one of the highest expressers of this protein together with muscle, the gastrointestinal tract, kidneys and male reproductive tissue (Figure 6$)(27,28)$.

As regards its cellular location, PCYOX1 seems to be mainly found in lysosomes (18), although it can also be exported to plasma lipoproteins secreted by the liver, such as VLDL and its metabolic product LDL (9). Interesting hypotheses may emerge in the future regarding this hepatic lysosomal presence as a result of recycling of LDL particles in the endolysosomal compartment or a direct movement of the enzyme to this organelle. These processes need to be studied in depth.

\subsection{Post-translational modifications (PTMs)}

The discrepancy between the theoretical prediction for a 505 -amino acid protein $(56 \mathrm{kDa})$ and the observed molecular mass $(63 \mathrm{kDa})$ could be due to glycosylation, as was proved using lectin-affinity chromatography in brain (29). Furthermore, a post-translational $\mathrm{N}$-glycosylation in plasma could also take place, as evidenced by digestion with peptide-N-glycosidase $\mathrm{F}$ (30). In fact, computational analyses revealed the existence of several motifs capable of being GalNAc O- or N-Glycosylated, as reflected in Table 4 . When recombinant prenylcysteine lyase was produced, analysis of both the recombinant and native enzymes revealed that the enzyme was glycosylated and also contained a signal peptide that was cleaved during processing (18). 
Glycosylation is not the only post-translational modification that this protein can undergo. Using different bioinformatics tools, we have predicted the existence of additional motifs susceptible of undergoing PTMs (acetylation, phosphorylation, nitrosylation, palmitoylation, succinylation, sumoylation and ubiquitination). A summary of these can be found in Table 4, and most are located on the prenylcysteine lyase domain (Figure 7). However, experimental confirmation of these modifications is lacking and their relevance as regards LDL activity needs to be addressed in the future.

\subsection{Protein structure and function}

PCYOX1 has three domains: an N-terminal signal peptide of 23 amino acids, an NAD(P)-binding Rossmann-like region (amino acids 39-106) and a prenylcysteine lyase domain (amino acids 128-496), as represented in Figure 7. The latter two are believed to exert the redox activity (31). Indeed, mutagenesis of Y455, E468 and M469 by alanines resulted in a loss of activity. These residues are preserved between humans and mice, thus suggesting that they are critical to the action of this enzyme in different species.

As it is a flavin adenine dinucleotide (FAD)-dependent thioether monooxygenase, PCYOX1 does not require NADPH as cofactor to catalyze S-oxidation of thioethers, in contrast to other enzymes such as cytochrome P450- and flavin-containing monooxygenases. Both farnesylcysteine and geranylgeranylcysteine could be its substrates, although Vmax has been reported to be twice as high with the former (6). However, prenylcysteine substrates must possess a free amino group since $\mathrm{N}$-acetylated prenylcysteines and prenyl peptides are not used by PCYOX1 (6). As shown in Figure 8, it cleaves prenylcysteines to cysteine and a $\mathrm{C}-1$ aldehyde of the isoprenoid moiety (farnesal in the case of $\mathrm{C}-15$ ). The enzyme utilizes a noncovalently bound flavin cofactor, requires molecular oxygen and releases hydrogen peroxide (7). The reaction proceeds by way of a sequential stereospecific mechanism in which the pro-S hydride of the farnesylcysteine is transferred to FAD to initiate the reaction and cysteine production is one of the primary rate-limiting steps in the process (33). In this way, the metabolic challenge of the degradation of prenylated proteins is solved by breaking the stable thioether bond of the modified cysteine (34). However, the release of hydrogen peroxide is a source of oxidative stress that may enhance damage in situations with increased enzyme activity.

\subsection{Protein interactions}

PCYOX1, as an enzyme, interacts with a vast number of different proteins and plays an important role in various cell processes. Although the methods used to discover these interactions (co-sedimentation, anti-tag co-immunoprecipitation or yeasttwo-hybrid) do not allow us to unveil its biological role $(11,35-37)$, this can be inferred by determining the characteristics of the proteins with which PCYOX1 interacts, as reflected in Table 5. Thus, it can participate in modulating the respiratory chain, cell death, cell signaling, movement and transport, metabolism and protein degradation. In fact, overexpression of PCYOX1 in COS7 cells has been found to be particularly cytotoxic (38). Its interaction with other chaperones is especially remarkable, with PCYOX1 acting as a co-chaperone or needing them for correct folding. Along the same lines, it should be pointed out that the interaction of PCYOX1 with the ubiquitination machinery and predicted ubiquitination sites could represent a means of controlling the half-life of PCYOX1.

Although not much is known about the involvement of PCYOX1 in disease processes, some of these interactions may suggest that it plays an important role in diseases such as cancer, due to the role of numerous proteins in important aspects of cell life. For instance, dysregulation of PCYOX1 may affect cell death via the CLU as a possible previously unexplored cause of cancer. In addition, a failure to interact with NDUFV2 could modulate the phenotypes of mitochondrial or other diseases in which this organelle has a profound influence. An important effort is therefore required to determine the influence of the high variability of PCYOX1, described in Table 1, on its ability to interact with other proteins and the putative consequences of these potential failures.

PCYOX1 can also be regulated by a large number of compounds (Table 6) (39) that can interact directly with the protein. In fact, docking assays carried out with the amino acid residues 36-70 of PCYOX1, which includes the majority of the NADP-binding domain as a target in well-conserved $2 \mathrm{GV} 8$, a crystalized protein, and three ligands (cyclosporine A, rosiglitazone and paracetamol), revealed that some regions are able to interact (40). These regions might form part of the active or regulatory sites. However, this would require crystallization of PCYOX1 and further folding, activity and stability studies to support this hypothesis.

\section{PCYOX1 IN PHYSIOLOGY AND DISEASE}

\subsection{Lipoproteins}

As indicated in the introduction, proteomic technologies using 2-D electrophoresis and MALDI-TOF/TOF analyses have confirmed the presence of PCYOX1 in lipoproteins in healthy volunteers. Two isoforms were described as new VLDLassociated proteins (9). Using liquid-phase isoelectrofocusing of LDL proteins, prenylcysteine lyase (PCL1), as named by the authors at the time, was also found to be a component of these lipoproteins, and their incubation with farnesylcysteine showed that the enzyme was active, releasing hydrogen peroxide. Banfi et al. showed that VLDL displayed a higher activity than LDL or HDL. Further support for this activity was obtained by proving that farnesol, a PCL1 inhibitor, decreased hydrogen peroxide generation by VLDL. These authors proved that the enzyme was carried in nascent lipoproteins secreted by HepG2 and that a 
second ultracentrifugation was unable to remove it. These facts indicate that the enzyme is a constitutive component of LDL (5), and therefore that LDLs are able to generate the potent oxidant hydrogen peroxide as a result. In an independent study, the presence of PCYOX1 protein was analyzed in VLDLs and LDLs purified from normolipidemic pooled plasmas by ultracentrifugation in $\mathrm{NaBr}$ or iodixanol gradients and proteomic analysis. Both fractions were found to contain the same amount, and the PCYOX1 levels correlated positively with those of APOA1 (41). Using a large number of subjects (230 volunteers), Dasthy et al. also prepared VLDLs and LDLs by ultracentrifugation and analyzed their constituent proteins by nano liquid chromatography-tandem mass spectrometry, confirming that PCYOX1 was associated with both VLDLs and LDLs (42). In a proteomic analysis of electronegative LDLs from normolipidemic subjects, no PCYOX1 was reported (43). No report has been found for Lp(a) and PCYOX1. Taken together, these observations indicate that PCYOX1 is a component of VLDLs and LDLs, its origin is hepatic, and the $\mathrm{H}_{2} \mathrm{O}_{2}$-generating role of this enzyme in LDLs will require more attention in future research as regards the role of atherogenic lipoproteins in atherogenesis. Equally, more research is needed to characterize specific LDL subtypes and the role of this enzyme in physiology and disease.

\subsection{Pcyox1-deficient mice}

To establish the importance of this enzyme at a biochemical or physiological level, Beigneux et al. created Pclydeficient mice. As expected, the absence of enzyme resulted in an accumulation of both farnesylcysteine and geranylgeranylcysteine in the brain and the liver, with no further pathological consequences, in thirty tissues from 4- and 10month-old Pcly-deficient mice analyzed using routine histologic stains (32). No observation of liver by electron microscopy or fluorescence to detect the accumulation of fluorescent material, which is usually observed in other lysosomal storage diseases, was carried out. Blood chemistry, including calcium, phosphate, glucose, cholesterol, triglycerides, alanine aminotransferase, aspartate aminotransferase, and creatinine, was normal in these mice, and serum lipid levels in the Pcly-deficient and wild-type mice did not differ on a high-fat diet either. The authors raised the possibility that the paralogous gene, Pcyox 1 , could compensate for the absence of PCYOX1, thus explaining why such a benign phenotype was observed, but this aspect was not explored further. At that time the enzyme was not suspected to be carried in LDLs, therefore no analysis was carried out in this regard. Therefore, characterization of these mice in terms of LDL oxidative properties and atherosclerosis development is a gap that still needs to be filled.

\subsection{PCYOX1 and the liver}

Mice lacking interstitial cells of Cajal (Sl/Sld and $\mathrm{W}(\mathrm{LacZ}) / \mathrm{Wv})$, the so-called pacemaker cells of the gut, showed significantly lower expression of hepatic Pcyox1 compared to wild-type mice (38). In a study of its nutritional regulation, our group observed no significant changes in hepatic Pcyox1 expression after the consumption of a nut-containing diet in Apoeknockout mice (24) or following squalene administration (25). However, the changes observed following depletion of N-3 polyunsaturated fatty acids (Table 3 ) indicate that some nutritional regulation may exist.

\subsection{PCYOX1 gene and cancer}

Next-generation sequencing of tumors has shown a large number of mutations in this gene (Figure 9), with the last exon being the DNA segment that accumulates the highest ratio of mutations in the tumors analyzed (44). However, no association studies have linked cancer to PCYOX1 gene variations to date (16), or to the protein variations considered. Despite this, different changes in gene expression have been observed in several tumors, as can be seen from Table 7. In this regard, pancreatic adenocarcinoma and osteosarcoma showed the highest increases, followed by large cell lung carcinoma, adenocarcinoma of the prostate, poorly differentiated hypernephroma, acute lymphoblastic leukemia, renal adenocarcinoma, ovarian cancer, adenocarcinoma of the colon, breast cancer, adenocarcinoma of the lung, and melanoma. In contrast, malignant mesothelioma showed the highest decrease, followed by pancreatic ductal adenocarcinoma, ovarian cancer, medulloblastoma, fibroadenoma, vulvar intraepithelial neoplasia, and clear cell renal carcinoma. In light of these findings, further studies are required to establish the role of this protein in this field and, as a consequence, of the catabolism of prenylated proteins in tumor development and evolution. The increase in hydrogen peroxide, and the consequent oxidative stress that its elevated activity may produce, should also be studied.

\subsection{PCYOX1 and neurodegenerative diseases}

Prenylcysteine lyase (PCYOX1) was found amongst the major glycoproteins from brains obtained from necropsies of Alzheimer's patients by using lectin-affinity and ion-exchange chromatographic analyses, followed by further separation using SDS-polyacrylamide gel electrophoresis (29). However, more experimental work is needed to determine the significance of this finding in this disease.

\subsection{PCYOX1 and obesity}

In an effort to investigate the differential gene expression of visceral and subcutaneous fat depots, Poussin et al. fed C57Bl/6 mice a high-fat diet for 6 months and mice with different body weights but similar levels of glucose intolerance were then treated with vehicle or rimonabant for one month to normalize body weight. Although not reported by the authors, we looked for the gene in GEO using deposition number GSE11790. An increased expression of Pcyox1 mRNA was observed in visceral but not in subcutaneous adipose tissue of mice receiving the high-fat diet. This increase was corrected following rimonabant administration (45). A hypothetical consequence of this increase would be an additional source of hydrogen peroxide and cellular oxidative stress. This enhanced oxidative stress in the visceral depot might explain the association of this adipose 
tissue with several complications of obesity. This interesting aspect requires further confirmation with new experiments and analyses of this enzyme in this particular setting with important repercussions in cardiovascular diseases.

\section{CONCLUSIONS}

The presence of PCYOX1 in low-density lipoproteins may aggravate LDL atherogenicity. In this regard, PCYOX1 acts as an enzyme that hydrolyzes prenylcysteines to cysteine and a C-1 aldehyde of the isoprenoid moiety. The enzyme utilizes a noncovalently bound flavin cofactor, requires molecular oxygen, and releases hydrogen peroxide. Two hypothetical situations may be envisioned in atherogenesis: first, the presence of LDLs carrying PCYOX1 in the subendothelial space may be more atherogenic since they can catabolize the final products of prenylcysteine-containing proteins, releasing the isoprenoid aldehyde and hydrogen peroxide. Second, these two products, which are not conveniently neutralized, may complicate the scenario, with the former possibly modifying the lysine groups of proteins, thus rendering them non-functional, and the second may increase oxidative damage. This would help to propagate cell damage following a primary insult in which these PCYOX1-containing LDLs are recruited. New research is therefore needed to determine whether this enzyme is particularly present in electronegative LDLs or in Lp(a). Since the liver is the primary source of this enzyme, new endeavors should be directed towards characterizing the hepatic regulation thereof and its translation into LDL changes. Moreover, many of the genetic variants should be characterized as regards this enzyme activity. Simplified assays should be developed to establish the role of the enzyme as a biomarker in cardiovascular disease. Based on the above, this protein is expected to play an important role in vascular disease in the future.

\section{ACKNOWLEDGMENTS}

We would like to thank Dr. Andrew Frankland for his assistance with manuscript editing. The work of this group was supported by grants from the Spanish Ministerio de Economía y Competitividad - European Regional Development Fund (201341651-R and 2016-75441-R) and the European Social Fund-Gobierno de Aragón (B-69). CIBER de Fisiopatología de la Obesidad y Nutrición (CIBEROBN) is an initiative of the Instituto de Salud Carlos III, Spain.

\section{REFERENCES}

1. Joseph L. Goldstein and Michael S. Brown: A Century of Cholesterol and Coronaries: From Plaques to Genes to Statins. Cell, 161(1), 161-172 (2015)

2. B. A. Ference, H. N. Ginsberg, I. Graham, K. K. Ray, C. J. Packard, E. Bruckert, R. A. Hegele, R. M. Krauss, F. J. Raal, H. Schunkert, G. F. Watts, J. Boren, S. Fazio, J. D. Horton, L. Masana, S. J. Nicholls, B. G. Nordestgaard, B. van de Sluis, M. R. Taskinen, L. Tokgozoglu, U. Landmesser, U. Laufs, O. Wiklund, J. K. Stock, M. J. Chapman and A. L. Catapano: Low-density lipoproteins cause atherosclerotic cardiovascular disease. 1. Evidence from genetic, epidemiologic, and clinical studies. A consensus statement from the European Atherosclerosis Society Consensus Panel. Eur Heart J (2017)

3. P. S. Jellinger, Y. Handelsman, P. D. Rosenblit, Z. T. Bloomgarden, V. A. Fonseca, A. J. Garber, G. Grunberger, C. K. Guerin, D. S. H. Bell, J. I. Mechanick, R. Pessah-Pollack, K. Wyne, D. Smith, E. A. Brinton, S. Fazio and M. Davidson: American Association of Clinical Endocrinologists and American College of Endocrinology Guidelines for Management of Dyslipidemia and Prevention of Cardiovascular Disease. Endocr Pract, 23(Suppl 2), 1-87 (2017)

4. P. Davidsson, J. Hulthe, B. Fagerberg, B. M. Olsson, C. Hallberg, B. Dahllof and G. Camejo: A proteomic study of the apolipoproteins in LDL subclasses in patients with the metabolic syndrome and type 2 diabetes. J Lipid Res, 46(9), 1999-2006 (2005)

5. C. Banfi, M. Brioschi, S. Barcella, R. Wait, S. Begum, S. Galli, A. Rizzi and E. Tremoli: Proteomic analysis of human lowdensity lipoprotein reveals the presence of prenylcysteine lyase, a hydrogen peroxide-generating enzyme. Proteomics, 9(5), $1344-$ $52(2009)$

6. L. Zhang, W. R. Tschantz and P. J. Casey: Isolation and characterization of a prenylcysteine lyase from bovine brain. J Biol Chem, 272(37), 23354-9 (1997)

7. W. R. Tschantz, J. A. Digits, H. J. Pyun, R. M. Coates and P. J. Casey: Lysosomal prenylcysteine lyase is a FAD-dependent thioether oxidase. J Biol Chem, 276(4), 2321-4 (2001)

8. IUBMB Enzyme Nomenclature, http://www.chem.qmul.ac.uk/iubmb/enzyme/EC1/8/3/5.html (2017)

9. C. Mancone, L. Amicone, G. M. Fimia, E. Bravo, M. Piacentini, M. Tripodi and T. Alonzi: Proteomic analysis of human very low-density lipoprotein by two-dimensional gel electrophoresis and MALDI-TOF/TOF. Proteomics, 7(1), 143-154 (2007) 
10. D. Moher, A. Liberati, J. Tetzlaff, D. G. Altman and P. G. The: Preferred Reporting Items for Systematic Reviews and MetaAnalyses: The PRISMA Statement. PLoS Med, 6(7), e1000097 (2009)

11. The Weizmann Institute of Science: Prenylcysteine Oxidase 1, 2017(January 15 2017), http://www.genecards.org/cgibin/carddisp.pl?gene=PCYOX1\&Key Words=PCYOX1 (2017)

12. EMBL-EBI: $\quad$ Ensembl release $\quad 87, \quad 2017$ (January $15 \quad$ 15 http://www.ensembl.org/Homo_sapiens/Gene/Summary?db=core;g=ENSG00000116005;r=2:70257386-70281191 (2017)

13. Genatlas, 2017, http://genatlas.medecine.univ-paris5.fr/fiche.php?onglet=1\&n=28551 (2017)

14. GTEX, http://www.gtexportal.org/home/gene/ENSG00000116005.7. (2017)

15. M. Lek, K. J. Karczewski, E. V. Minikel, K. E. Samocha, E. Banks, T. Fennell, A. H. O’Donnell-Luria, J. S. Ware, A. J. Hill, B. B. Cummings, T. Tukiainen, D. P. Birnbaum, J. A. Kosmicki, L. E. Duncan, K. Estrada, F. Zhao, J. Zou, E. Pierce-Hoffman, J. Berghout, D. N. Cooper, N. Deflaux, M. DePristo, R. Do, J. Flannick, M. Fromer, L. Gauthier, J. Goldstein, N. Gupta, D. Howrigan, A. Kiezun, M. I. Kurki, A. L. Moonshine, P. Natarajan, L. Orozco, G. M. Peloso, R. Poplin, M. A. Rivas, V. RuanoRubio, S. A. Rose, D. M. Ruderfer, K. Shakir, P. D. Stenson, C. Stevens, B. P. Thomas, G. Tiao, M. T. Tusie-Luna, B. Weisburd, H.-H. Won, D. Yu, D. M. Altshuler, D. Ardissino, M. Boehnke, J. Danesh, S. Donnelly, R. Elosua, J. C. Florez, S. B. Gabriel, G. Getz, S. J. Glatt, C. M. Hultman, S. Kathiresan, M. Laakso, S. McCarroll, M. I. McCarthy, D. McGovern, R. McPherson, B. M. Neale, A. Palotie, S. M. Purcell, D. Saleheen, J. M. Scharf, P. Sklar, P. F. Sullivan, J. Tuomilehto, M. T. Tsuang, H. C. Watkins, J. G. Wilson, M. J. Daly, D. G. MacArthur and C. Exome Aggregation: Analysis of protein-coding genetic variation in 60,706 humans. Nature, 536(7616), 285-291 (2016)

16. dbSNP: Short genetic variations, 2017, https://www.ncbi.nlm.nih.gov/snp (2017)

17. Vega, http://vega.sanger.ac.uk/Homo_sapiens/Location/View?db=core;g=OTTHUMG00000129671;r=2:7025495570287450;t=OTTHUMT00000331596 (2017)

18. W. R. Tschantz, L. Zhang and P. J. Casey: Cloning, expression, and cellular localization of a human prenylcysteine lyase. $J$ Biol Chem, 274(50), 35802-8 (1999)

19. Eukaryotic Promoter Database: (20 February 2017), http://epd.vital-it.ch/cgibin/get_doc?db=hgEpdNew\&format $=$ genome\&entry=PCYOX1_1 (2017)

20. UCSC Genome: (14 February 2017), http://genome.ucsc.edu/cgibin/hgTracks?db=hg19\&lastVirtModeType=default\&lastVirtModeExtraState=\&virtModeType $=$ default\&virtMode $=0$ \&nonVirtP osition $=\&$ position $=$ chr2\%3A70485231\%2D70508317\&hgsid=579348665_PFp0P9ddcQOCpdJtbiNxIHKs4GkX (2017)

21. The Encode Project Consortium: An Integrated Encyclopedia of DNA Elements in the Human Genome. Nature, 489(7414), 57-74 (2012)

22. Y. H. Zhoy: Atlas of Genetics and Cytogenetics in Oncology and Haematology, (13 February 2017), http://atlasgeneticsoncology.org/ (2017)

23. GEO profiles, https://www.ncbi.nlm.nih.gov/geoprofiles (2017)

24. J. C. Surra, C. Barranquero, M. P. Torcal, I. Orman, J. C. Segovia, N. Guillen, M. A. Navarro, C. Arnal and J. Osada: In comparison with palm oil, dietary nut supplementation delays the progression of atherosclerotic lesions in female apoE-deficient mice. Br J Nutr, 109(2), 202-9 (2013)

25. C. Gabas-Rivera, C. Barranquero, R. Martinez-Beamonte, M. A. Navarro, J. C. Surra and J. Osada: Dietary squalene increases high density lipoprotein-cholesterol and paraoxonase 1 and decreases oxidative stress in mice. PLoS One, 9(8), e104224 (2014)

26. The Jackson Laboratory: (18 February 2017), http://www.informatics.jax.org/marker/MGI:1914131 (2017)

27. M. Uhlen, L. Fagerberg, B. M. Hallstrom, C. Lindskog, P. Oksvold, A. Mardinoglu, A. Sivertsson, C. Kampf, E. Sjostedt, A. Asplund, I. Olsson, K. Edlund, E. Lundberg, S. Navani, C. A. Szigyarto, J. Odeberg, D. Djureinovic, J. O. Takanen, S. Hober, T. Alm, P. H. Edqvist, H. Berling, H. Tegel, J. Mulder, J. Rockberg, P. Nilsson, J. M. Schwenk, M. Hamsten, K. von Feilitzen, M. Forsberg, L. Persson, F. Johansson, M. Zwahlen, G. von Heijne, J. Nielsen and F. Ponten: Proteomics. Tissue-based map of the human proteome. Science, 347(6220), 1260419 (2015) 
28. The Human Protein Atlas project, 2017(18 February 2017), http://www.proteinatlas.org/ENSG00000116005-PCYOX1/tissue (2017)

29. Y. Huang, H. Tanimukai, F. Liu, K. Iqbal, I. Grundke-Iqbal and C. X. Gong: Elevation of the level and activity of acid ceramidase in Alzheimer's disease brain. Eur J Neurosci, 20(12), 3489-97 (2004)

30. T. Liu, W.-J. Qian, M. A. Gritsenko, D. G. Camp, M. E. Monroe, R. J. Moore and R. D. Smith: Human Plasma NGlycoproteome Analysis by Immunoaffinity Subtraction, Hydrazide Chemistry, and Mass Spectrometry. Journal of Proteome Research, 4(6), 2070-2080 (2005)

31. R. D. Finn, A. Bateman, J. Clements, P. Coggill, R. Y. Eberhardt, S. R. Eddy, A. Heger, K. Hetherington, L. Holm, J. Mistry, E. L. L. Sonnhammer, J. Tate and M. Punta: The Pfam protein families database, 2017(18 February 2017), http://pfam.xfam.org/protein/Q9UHG3 (2017)

32. A. Beigneux, S. K. Withycombe, J. A. Digits, W. R. Tschantz, C. A. Weinbaum, S. M. Griffey, M. Bergo, P. J. Casey and S. G. Young: Prenylcysteine lyase deficiency in mice results in the accumulation of farnesylcysteine and geranylgeranylcysteine in brain and liver. J Biol Chem, 277(41), 38358-63 (2002)

33. J. A. Digits, H. J. Pyun, R. M. Coates and P. J. Casey: Stereospecificity and kinetic mechanism of human prenylcysteine lyase, an unusual thioether oxidase. J Biol Chem, 277(43), 41086-93 (2002)

34. J. Y. Lu and S. L. Hofmann: Thematic review series: lipid posttranslational modifications. Lysosomal metabolism of lipidmodified proteins. J Lipid Res, 47(7), 1352-7 (2006)

35. C. Stark, B. J. Breitkreutz, T. Reguly, L. Boucher, A. Breitkreutz and M. Tyers: Biogrid: A General Repository for Interaction Datasets. Nucleic Acids Res, 34, D535-9 (2006)

36. STRING 10: STRING - Known and Predicted Protein-Protein Interactions, 2017(18 February), http://stringdb.org/cgi/network.pl?taskId=cI8IyoIjFLTF

37. Edward L. Huttlin, L. Ting, Raphael J. Bruckner, F. Gebreab, Melanie P. Gygi, J. Szpyt, S. Tam, G. Zarraga, G. Colby, K. Baltier, R. Dong, V. Guarani, Laura P. Vaites, A. Ordureau, R. Rad, Brian K. Erickson, M. Wühr, J. Chick, B. Zhai, D. Kolippakkam, J. Mintseris, Robert A. Obar, T. Harris, S. Artavanis-Tsakonas, Mathew E. Sowa, P. De Camilli, Joao A. Paulo, J. W. Harper and Steven P. Gygi: The BioPlex Network: A Systematic Exploration of the Human Interactome. Cell, 162(2), 425$440(2015)$

38. M. M. Wouters, J. M. Neefs, A. Kerchove d'Exaerde, J. M. Vanderwinden and K. A. Smans: Downregulation of two novel genes in Sl/Sld and W(LacZ)/Wv mouse jejunum. Biochem Biophys Res Commun, 346(2), 491-500 (2006)

39. Medical College of Wisconsin: Rat resource center, (13 February 2017), http://rgd.mcw.edu/ (2017)

40. A. Grosdidier, V. Zoete and O. Michielin: SwissDock, a protein-small molecule docking web service based on EADock DSS. Nucleic Acids Research, 39(Web Server issue), W270-W277 (2011)

41. H. Y. Sun, S. F. Chen, M. D. Lai, T. T. Chang, T. L. Chen, P. Y. Li, D. B. Shieh and K. C. Young: Comparative proteomic profiling of plasma very-low-density and low-density lipoproteins. Clin Chim Acta, 411(5-6), 336-44 (2010)

42. M. Dashty, M. M. Motazacker, J. Levels, M. de Vries, M. Mahmoudi, M. P. Peppelenbosch and F. Rezaee: Proteome of human plasma very low-density lipoprotein and low-density lipoprotein exhibits a link with coagulation and lipid metabolism. Thromb Haemost, 111(3), 518-30 (2014)

43. C. Bancells, F. Canals, S. Benítez, N. Colomé, J. Julve, J. Ordóñez-Llanos and J. L. Sánchez-Quesada: Proteomic analysis of electronegative low-density lipoprotein. Journal of Lipid Research, 51(12), 3508-3515 (2010)

44. Wellcome Trust $\quad$ Sanger $\quad$ Institute: $\quad(22 \quad$ February $\quad$ 2017), http://cancer.sanger.ac.uk/cosmic/cnv/details?coords=AA\%3AAA\&cnv=gain\&chr=2\&dr=\&end=70277392\&ln=PCYOX1\&gd= \&all_data=\&seqlen=506\&id=55983\&start=70258165 (2017)

45. C. Poussin, D. Hall, K. Minehira, A.-M. Galzin, D. Tarussio and B. Thorens: Different Transcriptional Control of Metabolism and Extracellular Matrix in Visceral and Subcutaneous Fat of Obese and Rimonabant Treated Mice. PLOS ONE, 3(10), e3385 (2008) 
46. GEO encode, https://www.ncbi.nlm.nih.gov/genome/gdv/?context=GEO\&acc=GSE31477 (2017)

47.

Protein

Data

Bank, http://www.rcsb.org/pdb/protein/Q9UHG3?evtc=Suggest\&evta=ProteinFeatureView\&evtl=autosearch_SearchBar_querySuggest (2017)

48. D. Karolchik, G. P. Barber, J. Casper, H. Clawson, M. S. Cline, M. Diekhans, T. R. Dreszer, P. A. Fujita, L. Guruvadoo, M. Haeussler, R. A. Harte, S. Heitner, A. S. Hinrichs, K. Learned, B. T. Lee, C. H. Li, B. J. Raney, B. Rhead, K. R. Rosenbloom, C. A. Sloan, M. L. Speir, A. S. Zweig, D. Haussler, R. M. Kuhn and W. J. Kent: The UCSC Genome Browser database: 2014 update. Nucleic Acids Research., 42, 764-70 (2014)

49. R. Gupta, E. Jung, A. A. Gooley, K. L. Williams, S. Brunak and J. Hansen: DictyOGlyc1.1.(18 February), http://www.cbs.dtu.dk/services/DictyOGlyc/ (2017)

50. C. Steentoft, S. Y. Vakhrushev, H. J. Joshi, Y. Kong, M. B. Vester-Christensen, K. T. Schjoldager, K. Lavrsen, S. Dabelsteen, N. B. Pedersen, L. Marcos-Silva, R. Gupta, E. P. Bennett, U. Mandel, S. Brunak, H. H. Wandall, S. B. Levery and H. Clausen: NetOGlyc 4.0. Server, 2017(18 February), http://www.cbs.dtu.dk/services/NetOGlyc/ (2017)

51. R. Gupta, E. Jung and S. Brunak: NetNGlyc 1.0. Server, 2017(18 February), http://www.cbs.dtu.dk/services/NetNGlyc/ (2017)

52. L. Kiemer, J. D. Bendtsen and N. Blom: NetAcet 1.0. Server, 2017(18 February), http://www.cbs.dtu.dk/services/NetAcet/ (2017)

53. F. Gnad, S. Ren, J. Cox, J. V. Olsen, B. Macek, M. Oroshi and M. Mann: PHOSIDA (phosphorylation site database): management, structural and evolutionary investigation, and prediction of phosphosites, 2017(18 February), http://141.6.1.1.02.1.8/phosida/index.aspx (2017)

54. K. Julenius: NetCGlyc 1.0., 2014(30 July), http://www.cbs.dtu.dk/services/NetCGlyc/ (2017)

55. N. Blom, S. Gammeltoft and S. Brunak: NetPhos 3.1. Server, 2017(18 February), http://www.cbs.dtu.dk/services/NetPhos/ (2017)

56. Phosphosite: (18 February 2017), http://www.phosphosite.org/proteinAction?id=18586\&showAllSites=true\#appletMsg (2017)

57. Y. Xu, J. Ding, L.-Y. Wu and K.-C. Chou: iSNO-PseAAC: Predict Cysteine S-Nitrosylation Sites in Proteins by Incorporating Position Specific Amino Acid Propensity into Pseudo Amino Acid Composition. PLoS ONE, 8(2), e55844 (2013)

58. J. Ren, L. Wen, X. Gao, C. Jin, Y. Xue and Y. X.: CSS-Palm 4.0., 2017(18 February), http://csspalm.biocuckoo.org/ (2017)

59. Q. Zhao, Y. Xie, Y. Zheng, S. Jiang, W. Liu, W. Mu, Y. Zhao, Y. Xue and J. Ren: GPS-SUMO 2.0.: a tool for the prediction of sumoylation sites and SUMO-interaction motifs. Nucleic Acids Research., 42, 325-330 (2014)

60. A. Li, X. Gao, J. Ren, C. Jin and Y. Xue: BDM-PUB: Computational Prediction of Protein Ubiquitination Sites with a Bayesian Discriminant Method, http://bdmpub.biocuckoo.org/prediction.php (2017)

61. Z. Chen, Y. Z. Chen, X. F. Wang, C. Wang, R. X. Yan and Z. Zhang: Prediction of Ubiquitination Sites by Using the Composition of k-Spaced Amino Acid Pairs. PLoS ONE, 6 (2011)

62. P. Radivojac, V. Vacic, C. Haynes, R. R. Cocklin, A. Mohan, J. W. Heyen, M. G. Goebl and L. M. Iakoucheva: Identification, Analysis and Prediction of Protein Ubiquitination Sites. Proteins: Structure, Function, and Bioinformatics. , 78, 365-380 (2010)

63. Expression atlas: $\quad(22 \quad$ February https://www.ebi.ac.uk/gxa/genes/ENSG00000116005?bs=\%7B $\% 22$ homo $\% 20$ sapiens $\% 22 \% 3 \mathrm{~A} \% 5 \mathrm{~B} \% 22 \mathrm{ORGANISM} \mathrm{PART} \% 2$ $2 \% 5 \mathrm{D} \% 7 \mathrm{D} \& \mathrm{ds}=\% 7 \mathrm{~B} \% 22 \mathrm{kingdom} \% 22 \% 3 \mathrm{~A} \% 5 \mathrm{~B} \% 22$ animals $\% 22 \% 5 \mathrm{D} \% 2 \mathrm{C} \% 22$ factors $\% 22 \% 3 \mathrm{~A} \% 5 \mathrm{~B} \% 22 \mathrm{disease} \% 22 \% 5 \mathrm{D} \% 7$ D\#differential (2017)

Abbreviations: prenylcysteine oxidase 1, PCYOX1; prenylcysteine lyase, PCL1 
Key Words: Prenylcysteine Oxidase 1, PCYOX1, 1200015P13Rik, Prenylcysteine Lyase 1, PCL1, Pcly, Review

Send correspondence to: Jesus Osada, Department of Biochemistry and Molecular Biology, Veterinary School, University of Zaragoza, Miguel Servet, 177, E-50013 Zaragoza, Spain, Tel: 34-976-761-644, Fax: 34-976-761-612, E-mail: Josada@unizar.es

Figure 1. Flow chart displaying the information collection process. Two different sources of data were used: data from online bioinformatics databases and a search in Pubmed. EndNote X7 (Thomson Reuters, New York, NY)

Figure 2. Gene expression for PCYOX1 in 53 different tissues from GTEx RNA-seq of 8555 samples (570 donors). Expression values are shown as $\log _{10}$ of RPKM (reads per kilobase of transcript per million mapped reads), calculated from a gene model with isoforms collapsed to a single gene. Box plots are shown as median and 25th and 75th percentiles (14).

Figure 3. Transcript (A) and protein (B) isoforms of PCYOX1 found in the liver using next-generation sequencing (14). The annotation is in accordance with that used in (17). Panel B shows the position of molecular markers.

Figure 4. Schematic representation of ENCODE information regarding regulatory elements of PCYOX1 gene. The presence of a $\mathrm{CpC}$ island of $1131 \mathrm{bp}$ and repetitive elements is shown.

Figure 5. Analysis of positional binding of transcription factors to the PCYOX1 gene in HepG2 cells. A: General overview of Genome Data viewer from the NCBI server (46) with information from ENCODE (21). Only transcription factors whose signals were higher than the input IgG from either rabbit or goat, which is considered to represent background noise, were selected. B: Detailed representation of the third intron region where transcription factors bind. GEO accession numbers are shown.

Figure 6. PCYOX1 protein and mRNA in different human tissues. Protein-expression scores are based on a best estimate of the "true" protein expression from a knowledge-based annotation. For genes where more than one antibody was used, a collective score was set displaying the estimated true protein expression. mRNA is expressed as number of transcripts per million reads. Reproduced with permission from (28).

Figure 7. Scheme showing PCYOX1 domains and distribution of post-translational modifications (PTMs) and putative secondary structure. A: the graphic shows the three domains, found between amino acids 1-23, 39-106 and 128-501 (31). B: location of PTM and prediction of secondary structure. In this representation, red denotes a potentially disordered region and blue a probably ordered region. In the hydropathy analysis, red represents hydrophobicity and blue hydrophilicity (47).

Figure 8. Reaction catalyzed by PCYOX1. Adapted from (7), reproduced with permission from the American Society for Biochemistry and Molecular Biology.

Figure 9. Distribution of mutations along the PCYOX1 gene found in tumor cells and the number of tumors analyzed to determine changes in this gene expression. Graphics generated using the Wellcome Trust Sanger Institute server (44).

Table 1. DNA changes observed in the coding region of the PCYOX1 gene in different human populations.

\begin{tabular}{|l|l|}
\hline Type of variation & Number found \\
\hline Coding sequence variant & 76 \\
\hline Frameshift variant & 16 \\
\hline In-frame deletion & 3 \\
\hline Mis-sense variant & 200 \\
\hline Mis-sense variant splice region variant & 4 \\
\hline Protein altering variant & 1 \\
\hline Splice region variant in coding sequence variant & 1 \\
\hline Start lost & 3 \\
\hline Stop gained & 15 \\
\hline Stop gained frameshift variant & 1 \\
\hline Synonymous variant & 1 \\
\hline A sume & 67 \\
\hline
\end{tabular}

A summary of protein variations found in Ensemble (12) and corresponding to the Exome Aggregation Consortium (15).

Table 2. Transcription factors found to control PCYOX1 gene expression in HepG2 $(14,21,48)$

\begin{tabular}{|l|l|l|}
\hline Transcription factor & Biology process & Tissue expression \\
\hline ARID3A & $\begin{array}{l}\text { Member of the ARID (AT-rich interaction domain) family involved in } \\
\text { cell cycle control, transcriptional regulation, and possibly in chromatin } \\
\text { structure }\end{array}$ & Ubiquitous expression \\
\hline BHLHE40 & $\begin{array}{l}\text { Basic helix-loop-helix protein believed to be involved in the control of } \\
\text { cell differentiation. }\end{array}$ & Ubiquitous expression \\
\hline CEBPB & CCAAT/enhancer-binding protein beta & Ubiquitous expression \\
\hline
\end{tabular}




\begin{tabular}{|l|l|l|}
\hline COREST & Demethylation of Lys-4 of histone H3 & Ubiquitous expression \\
\hline HNF4A & Nuclear transcription factor critical for liver development & Colon, ileum, kidney, liver, pancreas \\
\hline Max & $\begin{array}{l}\text { Proliferation and apoptosis thought H3 Lys-9 methyl-transferase } \\
\text { complex }\end{array}$ & Ubiquitous expression \\
\hline MAZ & Transcription factor involved in transcription initiation and termination & Ubiquitous expression \\
\hline SMC3 & Chromosome cohesion during cell cycle and in DNA repair & Ubiquitous expression \\
\hline
\end{tabular}

Table 3. Changes in hepatic Pcyox1 expression according to Genome Expressed Omnibus data bank and Array express.

\begin{tabular}{|c|c|c|}
\hline Experimental condition & Type of change & Accession number \\
\hline \multicolumn{3}{|l|}{ Genetic conditions } \\
\hline - Caspase-1 deficiency effect on fasted liver & Increased & GDS4922 \\
\hline $\begin{array}{l}\text { - NADH-cytochrome P450 reductase deletion effect on } \\
\text { the liver }\end{array}$ & Increased & GDS1349 \\
\hline $\begin{array}{l}\text { - Stearoyl-CoA desaturase 1-deficient mutants on a very } \\
\text { low-fat, high-carbohydrate diet }\end{array}$ & Increased & GDS1374 \\
\hline - SIRT1 deficiency effect on the liver & Increased & GDS3666 \\
\hline - Glycerol kinase knockout effect on liver & Increased & GDS1555 \\
\hline - Retinoblastoma protein deficiency effect on fetal livers & Increased & GDS2757 \\
\hline $\begin{array}{l}\text { - Mdr2 knockout model of hepatocellular carcinoma at } \\
\text { precancerous stages }\end{array}$ & Increased & GDS1990 \\
\hline - Livers with Dicer1 deficient hepatocytes & Increased & GDS3685 \\
\hline $\begin{array}{l}\text { - Lmna G609G knock-in model of Hutchinson-Gilford } \\
\text { Progeria Syndrome }\end{array}$ & Increased & GDS4490 \\
\hline $\begin{array}{l}\text { - LIGHT overexpressing } \mathrm{T} \text { cells and a high fat diet effect } \\
\text { on the liver }\end{array}$ & Increased & GDS3056 \\
\hline - Lipin 1-beta overexpression effect on the liver & Increased & GDS2291 \\
\hline $\begin{array}{l}\text { - Murine Pdss2 liver-specific knockouts as a model of } \\
\text { primary mitochondrial dysfunction }\end{array}$ & Decreased & GDS3454 \\
\hline $\begin{array}{l}\text { - IL-4 and IL-13 double mutant liver response to acute } \\
\text { injury }\end{array}$ & Decreased & GDS5073 \\
\hline $\begin{array}{l}\text { - Suppressor of cytokine signaling } 3 \text { deficiency effect on } \\
\text { the regenerating liver }\end{array}$ & Decreased & GDS3149 \\
\hline $\begin{array}{l}\text { - Hepatocyte nuclear factor } 4 \text { alpha knockout effect on the } \\
\text { embryonic liver }\end{array}$ & Decreased & GDS1916 \\
\hline $\begin{array}{l}\text { - Conditional GBA1 deletion model of Type } 1 \text { Gaucher } \\
\text { Disease }\end{array}$ & Decreased & GDS4162 \\
\hline \multicolumn{3}{|l|}{ Dietary conditions } \\
\hline $\begin{array}{l}\text { - N-3 polyunsaturated fatty acid depletion effect on the } \\
\text { liver }\end{array}$ & Increased & GDS4796 \\
\hline - Ketogenic diet effect on the liver & Increased & GDS2738 \\
\hline - Dietary palatinose effect on liver & Decreased & GDS5435 \\
\hline \multicolumn{3}{|l|}{ Pathological conditions } \\
\hline - Concanavalin A-induced fulminant hepatitis model & Increased & GDS3752 \\
\hline - Alcoholic hepatitis in Homo sapiens & Decreased & GDS4389 \\
\hline - Oxidative stress and Snell dwarf liver & Decreased & GDS683 \\
\hline - Pharmacological agents & & \\
\hline - Glucocorticoid effect on the female and male liver & Decreased & GDS5036 and 5035 \\
\hline $\begin{array}{l}\text { - High doses of perfluorooctanoic acid effect on fetal } \\
\text { liver }\end{array}$ & Decreased & GDS3410 \\
\hline $\begin{array}{l}\text { - Sex-dependent and growth hormone-dependent gene } \\
\text { expression in rat }\end{array}$ & Decreased & GDS862 \\
\hline
\end{tabular}

https://www.ncbi.nlm.nih.gov/geoprofiles and https://www.ebi.ac.uk/arrayexpress/ . Accessed on 12 February 2017

Table 4. Post-translational modifications of human PCYOX1 and participant amino acids

\begin{tabular}{|c|c|c|c|}
\hline Modification & Modified amino acids & $\begin{array}{l}\text { Prediction server } \\
\end{array}$ & References \\
\hline Glycosylation & No reports $(*)$ & DictyOGlyc & $(49)$ \\
\hline GalNAc O-glycosylation & Thr (398), Ser (322) & NetOGlyc & $(50)$ \\
\hline N-Glycosylation & Asn $(196,288,323,353)$ & NetNGlyc & (51) \\
\hline N-Glycosylation & Asn $(196,288,323,353)$ & NetNGlyc & $(51)$ \\
\hline Acetylation & Lys $(59,100,406)$ & $\begin{array}{l}\text { NetAcet } \\
\text { Phosida }\end{array}$ & $\begin{array}{l}(52) \\
(53)\end{array}$ \\
\hline Mannosylation & No reports $(* *)$ & NetCGlyc & (54) \\
\hline Phosphorylation & $\begin{array}{l}\text { Thr }(47,77,198,286,299,303,308,325,345,373,407,414,503) \text {, Ser }(11,24,90, \\
109,110,131,149,171,177,178,209,239,259,266,267,273,276,301,305,322, \\
358,382,409,419,435,465), \operatorname{Tyr}(51,85,174,191,297,308,339,403,425,427, \\
439,482,498)\end{array}$ & $\begin{array}{l}\text { NetPhos } \\
\text { Phosphosite Phosida }\end{array}$ & $\begin{array}{l}(55)(56) \\
(53)\end{array}$ \\
\hline
\end{tabular}




\begin{tabular}{|l|l|l|l|}
\hline S-nitrosylation & Cys $(24,242,258,445)$ & GPS-SNO \\
\hline Palmitoylation & Cys $(20,22,24)$ & CSS-Palm 4.0. \\
\hline Succinylation & Lys $(415,430)$ & $(57)$ \\
\hline Sumoylation & Lys $(62,349,502)$ & GPS-SUMO 2.0. \\
\hline & Lys $(59,100,104,181,266,500,502)$ Lys $(56,420)$ Lys (392) & BDM-PUB \\
Ubiquitination & & $\begin{array}{l}\text { CKSSAAP } \\
\text { Ubpred }\end{array}$ & $\begin{array}{l}(60)(63) \\
\text { 62) }\end{array}$ \\
\hline
\end{tabular}

*DictyOGlyc reports no results, so there are no probable glycosylation sites in the O-residues of PCYOX1. ** No results were found using NetCGlyc. Mannosylation is uncommon in mammal proteins. All predictions were made using bioinformatics tools that checked the homology of PCYOX1 protein domains with experimental databases for the PTMs. The sequence used to predict the PTMs of PCYOX1 was the canonical sequence of the isoform PCYOX1-001.

Table 5. Protein interactions of human PCYOX1 and the cell processes involved

\begin{tabular}{|l|l|}
\hline Proteins & Biology process \\
\hline ERP27, FKBP7, FKBP14 & Chaperones \\
\hline CLU & Cell death \\
\hline NENF, RHOBTB3, ANTXR1, PLAUR, DLK1, ISLR, TRAC & Cell signaling \\
\hline LAMP1, SLC9A3R1, UBAC2, TRDN & Cell transport \\
\hline DYNC2LI1 & Cell movement \\
\hline PBLD, FDPS, BLVRA, ENTPD4, PLTP & Metabolism \\
\hline NDUFV2 & Respiratory chain \\
\hline FOXRED2, UBC & Ubiquitination \\
\hline
\end{tabular}

These interactions were discovered by co-sedimentation, co-immunoprecipitation, and yeast-two hybrid system (11, 35-37).

Table 6. Regulation of PCYOX1 expression with different chemical compounds.

\begin{tabular}{|l|l|}
\hline Type of agents & Compounds \\
\hline Toxic agents & $\begin{array}{l}\text { 2,3,7,8-Tetrachlorodibenzodioxine, 4'-diaminodiphenylmethane benzopyrene, benzene, 2,6-dinitrotoluene, paraquat, bisphenol } \\
\text { A, aroclor 1254, crocidolite asbestos, } N \text {-methyl-4--phenylpyridinium, ammonium chloride, tributylstannane, 3-isobutyl-1-methyl- } \\
\text { 7H-xanthine, butanal, flutamide, silicon dioxide }\end{array}$ \\
\hline Toxins & Aflatoxin B1 \\
\hline Immunosuppressor & Cyclosporine A \\
\hline Antidiabetic & Rosiglitazone \\
\hline Adrenaline analogous & $3,4-$-Methylenedioxymethamphetamine \\
\hline ROS & tert-Butyl hydroperoxide, 4-hydroxynon-2-enal \\
\hline Antitumor & Methotrexate \\
\hline PPAR $\alpha$ & Pirinixic acid \\
\hline Non-steroid anti-inflammatory & Paracetamol \\
\hline Antibiotics & Gentamycin \\
\hline Anti-epilepsy drug & Valproic acid, phenytoin \\
\hline Metals & Zinc, nickel, copper(II) sulfate, titanium dioxide, lead diacetate, potassium dichromate \\
\hline Hormones & Testosterone enanthate, dexamethasone \\
\hline
\end{tabular}

Interactions between PCYOX1 and different organic and inorganic compounds as drugs and metabolites. All of them were experimentally discovered by different research groups $(22,23,39)$

Table 7. Changes of PCYOX1 gene expression in different tumors

\begin{tabular}{|l|l|}
\hline Type of tumor & Log $_{2}$-fold change \\
\hline Pancreatic adenocarcinoma & 3.1. \\
\hline Osteosarcoma & 3.1. \\
\hline Large cell lung carcinoma & 3 \\
\hline Adenocarcinoma prostate & 2.7. \\
\hline Poorly differentiated hypernephroma & 2.6. \\
\hline Acute lymphoblastic leukemia & 2.6. \\
\hline Renal adenocarcinoma & 2.5. \\
\hline Ovarian cancer & 2 \\
\hline Adenocarcinoma of colon & 1.9. \\
\hline Breast cancer & 1.9. \\
\hline Adenocarcinoma of lung & 1.6. \\
\hline Melanoma & 1.3. \\
\hline Clear cell renal carcinoma & -1.2. \\
\hline Vulvar intraepithelial neoplasia & -1.2. \\
\hline Fibroadenoma & -1.4. \\
\hline Medulloblastoma & -1.7. \\
\hline Ovarian cancer & -1.8. \\
\hline Pancreatic ductal adenocarcinoma & -1.9. \\
\hline Malignant mesothelioma & -2.5. \\
\hline A summary of da & \\
\hline
\end{tabular}

A summary of data found in EMBL-EBI (63) and Wellcome Trust Sanger Institute (44) servers

Running title: PCYOX1 and LDL 


\section{Publication processes}

This galley is provided to you for text correction. Please read this galley with great care and make all necessary text changes. The temporary manuscript number of this document is the number assigned to the galley that you received. This number is also included in the letter of acceptance that you received. List of changes to be made before the galley is submitted for publication is included below. Full details of formatting guideline is available online at http://www.bioscience.org/authors. A temporary manuscript number has been assigned to this manuscript. This number appears at the top of the letter of acceptance and is used to number the galley. Please use this number and name of the managing editor in any future correspondence to the Frontiers in Bioscience until the manuscript is published. The permanent manuscript number will be assigned upon publication of the manuscript. Submission of manuscript for publication entails the following steps:

1. Submission of necessary documents by Email. Please send all the required materials including the galley, doi.doc, forms.doc and figure files (fig1.jpg etc) to the editorial office of the Frontiers in Bioscience (fbs@bioscience.org). Please fill and return the publication forms by placement of name of authors on the copyright page, and selecting a package. Please note that submission of publication packages can be done during online submission of the manuscript.

1.1. Galley. Submit final revised galley. Do not change the manuscript number. Save revised file with the same name assigned to the file that you receive such as (4435.doc).

1.2. DOI.doc. Submit DOI linked references with tabular format and live links. Submit as doi.doc (do not use any other filename).

1.3. Figures. Submit final figures. Submit figures as jpg files named fig1, fig2 etc. Do not use any other style such as Fig1 or Fig 1 etc.

1.4. Publication forms. Publication forms including copyright and publication packages.

\subsection{Properly formatted galley}

Text. Please note that all manuscripts should have flawless English and should be free of spelling and scientific errors. Tables and figure legends (not figures) should be placed at the end of the manuscript.

- Change non-English characters to their English correlates in the title, abstract, names or affiliations.

Authors. Format per FBS style. No non-English characters. Provide full first and last name

Affiliations. Format per FBS style. No non-English characters

Title: Provide a title less than 75 characters with spaces.

Abstract. Format per FBS style. No non-English characters. Please reduce the number of words in the abstract to less than 175 words.

\section{Send correspondence to:}

Note: One corresponding author, address, tel, fax and Email should be included in this section. The corresponding author address should be the same as the one listed on the first title page and only one primary address will be included in PubMed. In the title section, the primary address should be assigned with a superscripted number as 1 such as follows

Corresponding author name ${ }^{1}$

${ }^{1}$ Corresponding author name

Address: Provide or check for accuracy

Tel: Provide or check for accuracy

Fax: Provide or check for accuracy

E-mail: Provide or check for accuracy

Running title. Provide a running title less than 55 characters. See sample (Running title: Production and reduction of ROS)

\subsection{Figure files}


Figures. Provide per FBS style. Figures should be of high quality and $>300 \mathrm{dpi}$. Crop the figure close to the image so that no white space around any figure is visible. Place multipart figures in a single file. Place labels in the left lower corner of each figure. Figures that do not follow the above guidelines can not be used for document processing.

Submit as fig1 as jpg file (.jpg is an extension of the file and not part of the name of the file), etc. Do not use any other filename such as fig 1 or Fig1 or Fig 1 etc. Do not include filename or any other name or any white or unused spaces around figure.

\subsection{DOI references with live links}

DOI linked references provided within a file named doi.doc. All doi linked references must have live links. Do not paste data as text. Paste in native format to maintain the links. Live links will lead to conversion of the cursor to a hand. See sample below. Place cursor over doi:10.1002/ijc.20631 and you will note the cursor changes to a hand. All doi in the ref list must have similar live links. Follow the steps provided below to obtain the doi with live links. Some references may not have doi. Please disregard such results. Such references will be followed by a statement such as (doi not found). Please do not remove such references from the list.

1. Go to https://apps.crossref.org/simpleTextQuery

2. Copy about 50 references at a time from the referfence list

3. Paste the references into the query box

4. Click "submit" button

5. The doi linked references will be displayed in about 30 seconds on the screen

6. Copy all the references including those that do not have live doi links by pressing "Control+C"

7. Paste the data into a blank new document

8. Repeat this process for other references

9. Save the file as doi.doc. Do not add any other text to the page (such as DOI references etc)

A reference sample with live doi.links is shown below

K Almholt, LR Lund, J Rygaard, BS Nielsen, K Danø, J Rømer, M Johnsen: Reduced metastasis of transgenic mammary cancer in urokinase-deficient mice. Int J Cancer 113 (4), 525-32 (2005).

doi:10.1002/ijc.20631

\subsection{Publication forms}

Invited Review: If your manuscript is an invited review, the basic publication charge for your manuscript is waived. Please choose the "Basic Package" in the order forms if you do not wish to receive a professional galley, reprint, PDF or subscription. Each package includes a series of options including professional galley, reprint, PDF and subscription as specified in the publication forms. Professional galley has a rich two column format with figures and tables inserted in the page where they are cited. As part of any of these packages, you will also receive a PDF file with a license for distribution. Please note that the published online documents do not carry such a license. Some packages also include hard copy reprints and subscription. If you would like to choose any of these packages, please mark them in the order and invoice forms.All these packages have reduced fees for invited reviews.

\section{Acknowledgements by the corresponding author}

I am the corresponding author of this manuscript and I acknowledge that

1. I have read this galley and have made all necessary text changes

2. I approve the publication of this galley without any further text changes.

3. If I wish to request any further changes not included in this galley, I will submit the reprocessing form (http://www.bioscience.org/authors).

Place your name here: [ Click and type ] Osada

Place your E-mail here: [ Click and type ] josada@unizar.es

Place date here: [ Click and type ] July $\mathbf{2 4}^{\text {th }}, \mathbf{2 0 1 7}$

\section{Authors and affiliations}


Authors. Format per FBS style. No non-English characters. Provide full first and last name

\begin{tabular}{|c|c|c|c|}
\hline First Name & Middle Name & Last Name & Affiliation \\
\hline Luis & V. & $\begin{array}{l}\text { Herrera- } \\
\text { Marcos }\end{array}$ & $\begin{array}{l}\text { Departamento de Bioquimica y Biologia Molecular y Celular, Facultad de } \\
\text { Veterinaria, Instituto de Investigacion Sanitaria de Aragon-Universidad de } \\
\text { Zaragoza, Zaragoza, E-50013, Spain, } \\
\text { Instituto Agroalimentario de Aragon, CITA-Universidad de Zaragoza, Spain }\end{array}$ \\
\hline Jose & M. & $\begin{array}{l}\text { Lou- } \\
\text { Bonafonte }\end{array}$ & $\begin{array}{l}\text { Instituto Agroalimentario de Aragon, CITA-Universidad de Zaragoza, Spain } \\
\text { Departamento de Farmacologia y Fisiologia, Facultad de Ciencias de la } \\
\text { Salud y del Deporte, Instituto de Investigacion Sanitaria de Aragon- } \\
\text { Universidad de Zaragoza, Huesca, E-22002, Spain } \\
\text { CIBER de Fisiopatologia de la Obesidad y Nutricion, Instituto de Salud } \\
\text { Carlos III, Madrid, E-28029, Spain }\end{array}$ \\
\hline Maria & V. & $\begin{array}{l}\text { Martinez- } \\
\text { Gracia }\end{array}$ & $\begin{array}{l}\text { CIBER de Fisiopatologia de la Obesidad y Nutricion, Instituto de Salud } \\
\text { Carlos III, Madrid, E-28029, Spain }\end{array}$ \\
\hline Carmen & & Arnal & $\begin{array}{l}\text { Instituto Agroalimentario de Aragon, CITA-Universidad de Zaragoza, Spain } \\
\text { CIBER de Fisiopatologia de la Obesidad y Nutricion, Instituto de Salud } \\
\text { Carlos III, Madrid, E-28029, Spain } \\
\text { Departamento de Patologia Animal, Facultad de Veterinaria, Instituto de } \\
\text { Investigacion Sanitaria de Aragon-Universidad de Zaragoza, Zaragoza, E- } \\
\text { 50013, Spain }\end{array}$ \\
\hline Maria & A. & Navarro & $\begin{array}{l}\text { Departamento de Bioquimica y Biologia Molecular y Celular, Facultad de } \\
\text { Veterinaria, Instituto de Investigacion Sanitaria de Aragon-Universidad de } \\
\text { Zaragoza, Zaragoza, E-50013, Spain, } \\
\text { Instituto Agroalimentario de Aragon, CITA-Universidad de Zaragoza, Spain } \\
\text { CIBER de Fisiopatologia de la Obesidad y Nutricion, Instituto de Salud } \\
\text { Carlos III, Madrid, E-28029, Spain }\end{array}$ \\
\hline Jesus & & Osada & $\begin{array}{l}\text { Departamento de Bioquimica y Biologia Molecular y Celular, Facultad de } \\
\text { Veterinaria, Instituto de Investigacion Sanitaria de Aragon-Universidad de } \\
\text { Zaragoza, Zaragoza, E-50013, Spain } \\
\text { Instituto Agroalimentario de Aragon, CITA-Universidad de Zaragoza, Spain } \\
\text { CIBER de Fisiopatologia de la Obesidad y Nutricion, Instituto de Salud } \\
\text { Carlos III, Madrid, E-28029, Spain }\end{array}$ \\
\hline & & & \\
\hline & & & \\
\hline & & & \\
\hline
\end{tabular}

Managing Editor: Ahmed Bakillah, Mohamed Zaiou 